\title{
Discrimination of time intervals bounded by tone bursts
}

\author{
PIERRE L. DIVENYI \\ Veterans Administration Medical Center, Martinez, California 94553 \\ and \\ RICHARD M. SACHS \\ Central Institute for the Deaf, St. Louis, Missouri 63110
}

\begin{abstract}
Trained listeners had to discriminate, in a four-level 2AFC paradigm, the duration of a time interval bounded by pairs of brief tone bursts. The time intervals ranged from 10 to $100 \mathrm{msec}$. When the tone-burst markers were similar in their intensity ( $86 \mathrm{~dB} \mathrm{SPL})$ and frequency $(1 \mathrm{kHz})$, the just noticeable time difference was found to be monotonically related to the base interval. On the other hand, when the intensity of the first marker was severely attenuated (by $50 \mathrm{~dB}$ ) or when a large (2-octave) difference was introduced between the frequencies of the two markers, the time discrimination functions became nonmonotonic. A similar, albeit slight, departure from monotonicity was also achieved by making the second marker much longer than the first (300 msec vs. $10 \mathrm{msec}$ ). The nonmonotonicity of these time discrimination functions is compared to the well-documented nonmonotonicity that may be observed for voice onset time (VOT) discrimination functions.
\end{abstract}

Auditory discrimination of brief unfilled (empty) time intervals has been shown to obey some psychophysical law, e.g., Weber's law': a temporal difference just noticeable by the observer has been found to be proportional to, or at least a monotonic function of, the time interval to be discriminated (Abel, 1972; Chistovich, 1959; Divenyi \& Danner, 1977). While this observation seems to hold true for time intervals marked by a wide variety of simple sounds, such as clicks, tone bursts, and noise bursts, there is an important class of auditory markers that represent an exception to this rule. Indeed, it is well known that auditory discrimination of time intervals defined by pairs of speech or speech-like sounds defies simple psychophysical laws: the threshold for the discrimination of such intervals is minimum in a certain (narrow) time range and increases for durations above and below this range. Such a nonmonotonicity of the just-discriminable time difference with respect to the base interval has mainly been associated with the perception of voice onset time (VOT), i.e., with

Portions of this paper were presented at the 92nd meeting of the Acoustical Society of America (San Diego, November 1976). The authors gratefully acknowledge the assistance of W. F. Danner and $\mathrm{K}$. Grant in conducting the experiments, and that of T. Hogan in analyzing the data. The experiments were performed at Central Institute for the Deaf. This research was supported by a grant from NINDS to Central Institute for the Deaf and by the Veterans Administration. Requests for reprints should be addressed to Research Department, Central Institute for the Deaf, 818 South Euclid, St. Louis, Missouri 63110. the onset of voicing that follows a stop consonant burst (Abramson \& Lisker, 1970; Samuel, 1977). However, the same type of nonmonotonicity has also been demonstrated to occur for the discrimination of intervals marked by a "speech-like" pair of sounds consisting of a short noise burst followed by a buzz (Divenyi \& Danner, 1975; Miller et al., 1976), or by the onsets of a pair of overlapping tones different in frequency (Pisoni, 1977).

Two main hypotheses have been proposed to account for the curious phenomenon that these nonmonotonic discrimination functions represent. The first of these is linguistic-oriented: it is based on the well known fact that the lowest time difference threshold obtained for the discrimination of VOT in stop consonant-vowel complexes sharing the same place of articulation coincides with the perceptual boundary for the voiced-voiceless distinction (Liberman et al., 1967). For example, the perceptual boundary for the distinction of $\mathrm{a} / \mathrm{ba} /$ and $\mathrm{a} / \mathrm{pa} /$ CV pair in English corresponds to a VOT of approximately 20-25 msec; it is also at an interval of similar duration that discrimination of two synthetic bilabial stop consonant-/a/-vowel pairs that differ in their VOT alone is optimum (Abramson \& Lisker, 1970). Such a correspondence signifies that two CV sounds, identical in every respect except for some fixed difference in their VOT, may be discriminated only if one of them falls in the "voiced" and the other in the "voiceless" perceptual category. Since perceptual category boundaries for the voiced-voiceless distinc- 
tion correspond reasonably well with articulatory category boundaries within a given language (Lisker \& Abramson, 1970), the existence of distinct perceptual categories may be thought to reflect learning of articulatory categories. Thus, the first hypothesis is a natural outgrowth of the motor theory of speech perception.

In contrast, the second hypothesis (Miller et al., 1976) is auditory-oriented: it attributes the departure of VOT discrimination from a monotonic psychophysical law to the likelihood of perceptual interactions that may take place between various components of a stimulus complex. In stimulus complexes where it is possible to distinguish two perceptually distinct successive elements, such as in speech sounds, shortening the temporal separation between the elements may lead to perceptual interactions which could modify the Gestalt of the whole complex. For example, one such modification could affect the perception of successiveness, especially when the two elements considered are dissimilar (Hirsh, 1959). Other kinds of modifications could lead to effects not unlike backward masking, especially when the second element is longer and more intense than the first (Elliott, 1971; Samoilova, 1960). It is understood that perceptual interactions of this kind will operate only in stimulus configurations in which the temporal separation of the two elements is small. Conversely, the effect of such interactions will become less and less important as the two successive elements become more and more remote in time. The critical temporal separation beyond which perceptual interference between the two markers is no longer noticeable will be the one at which the discrimination function for the interval between the elements, in general, and for VOT, in particular, will have a minimum. Thus, this second hypothesis follows a more strictly auditory theory of speech perception.

Results of a recent study (Divenyi \& Danner, 1977) tend to support this second hypothesis. In that study, it was demonstrated that discrimination of short (25-msec) time intervals marked by two brief $(20-\mathrm{msec})$ tone bursts deteriorates as a result of either decreasing the intensity of the first marker or increasing the frequency separation between the two markers. Thus, it appears that a stimulus complex consisting of a rapid succession of two tone bursts differing either in their intensity or in their frequency may give rise to a perceptual interaction of the type Miller et al. (1976) were referring to. It is, therefore, not surprising that time discrimination functions for some unequal-intensity and unequal-frequency conditions were found to be nonmonotonic in the Divenyi and Danner (1977) experiments.

However, that former study was not focused on the issue of discrimination functions and, therefore, did not comprise a systematic exploration of the stimulus parameters which could alter the normally monotonic shape of time discrimination functions. The objective of the present experiments was to examine in some detail the influence of a few selected stimulus parameters on the discrimination threshold of time intervals in the 10 - to $100-\mathrm{msec}$ range, i.e., in the range most important in VOT perception, with special attention devoted to the question of in what circumstances time discrimination functions may become nonmonotonic. In order to keep the stimulus as simple as possible, the time markers in these experiments were bursts of pure tones. The stimulus parameters investigated included difference in intensity, difference in frequency, and difference in duration between the two markers.

\section{PROCEDURES}

Thresholds for the discrimination of unfilled auditory time intervals were obtained by using a four-level 2AFC (two-alternative forced-choice) method. Although much of the VOT discrimination data reported in the speech literature was obtained using a different method (mostly ABX or "same-different"), a series of earlier experiments (Sachs \& Grant, 1976) showed that performance of trained subjects discriminating VOT in synthetic speech sounds was essentially identical, regardless of whether a 2 AFC or a "same-different" (i.e., AB) paradigm was employed. ${ }^{2}$ We adopted the $2 \mathrm{AFC}$ paradigm because it is criterion free (Green \& Swets, 1966).

In each trial, subjects were presented with two time intervals, each of which was defined by a pair of tone bursts. The duration of one interval was $t$, while that of the other was $t+\Delta t \mathrm{msec}$. In each block of 100 trials, only one base interval duration, $t$, was used, but the time difference $\Delta t$ could assume any one of four preselected values. These four values were chosen such that a given subject was correct about $55 \%-65 \%$ of the time when the time difference was the smallest, and about $85 \%-98 \%$ of the time when it was the largest in the set. Six base durations $t, 10,20,30$, 50,75 , and $100 \mathrm{msec}$ as measured from the onset of the first marker to the onset of the second, were investigated in each of the three experiments. The tone burst markers were gated at a positive-going zero crossing and had envelopes shaped with 2.5-msec rise and fall times. The first marker of each interval had a total duration of $10 \mathrm{msec}$, i.e., its plateau was $5 \mathrm{msec}$ long, whereas the second marker's duration was either fixed at $100 \mathrm{msec}$ (Experiments 1 and 2) or it was parametrically varied (Experiment 3 ). Because of the rise and fall times, the subjects heard two perceptually distinct markers in each interval, even when the duration of the interval was $10 \mathrm{msec}$, i.e., when the onset of the second marker immediately followed the offset of the first. The frequency of the markers was either held constant at $1 \mathrm{kHz}$ (Experiments 1 and 3) or it was variable (Experiment 2). The level of the markers (measured at the subject's earphone) was $86 \mathrm{~dB}$ SPL in all three experiments, with the exception of Experiment 1 in which the first marker of each interval was presented at various, attenuated levels. There was an intertrial interval of $600 \mathrm{msec}$ separating the first markers of the two intervals in each trial. An intertrial interval of such duration has been found to be sufficiently long to eliminate (or at least greatly reduce) the likelihood that the subjects could have based their judgments on the temporal separation between the end of the second marker of the first interval and the beginning of the first marker of the second interval (Penner, 1976). The trials were paced at a regular 3.2-sec cycle. 
A Wavetek Model 114 triggerable voltage-controlled oscillator generated the stimuli. The signal, after passing through an electronic switch (Grason-Stadler Model 1287B), was amplified, attenuated, and led to the left side of a pair of earphones (TDH 49 in MX 41/AR cushions embedded in Rudmose circumaural otocups). The subjects were run simultaneously and were seated in a single sound-treated chamber. Their task consisted of determining, by pressing one of two labeled keys, which one of the two intervals was longer in a given trial. Visual feedback followed each response. Sequencing of the stimuli and collection of the responses were accomplished by a set of Grason-Stadler 1200-series logic modules and by MELOGIC-II, a special-purpose digital device designed by the first author.

Three male, high-school age (15-17 years) subjects participated in the experiments and were paid hourly wages. At the beginning of the data collection, they had had at least 6 months of experience in other, similar auditory time discrimination experiments. To ensure data stability, a minimum of six blocks of 100 responses were collected in each experimental condition. Each observer's responses were pooled over all blocks of a given condition and a d' estimate was computed for each of the four $\Delta t$ time differences presented in that condition.

The experimental data were analyzed in two ways. First, in the conventional manner, we took several fixed $\Delta t$ values ("one-step," "two-step," etc.; see Liberman et al., 1967) and examined the subjects' performance level at these time differences over all base durations used. The results illustrated in Figure 1 reflect the outcome of such an analysis. However, as this figure plainly suggests, such a representation of the data is not as informative as we would have desired: in easy conditions, a given time difference, $\Delta t$, yielded percent correct scores that were too high to permit meaningful inferences, whereas in difficult conditions, the same $\Delta t$ resulted in percent correct scores that were too low. Therefore, we decided to analyze our data also in a second way-a way which is customary in psychophysical research but which, unfortunately, has not yet gained wide acceptance in speech perception studies. This manner of analysis is based on thresholds calculated from the psychometric functions obtained for each condition. In our particular case, we found that the four $\mathrm{d}^{\prime}$ vs. $\Delta t$ psychometric points could be well fitted with a straight line represented by the $\mathrm{d}^{\prime}=\mathrm{a}+\mathrm{b} \log \Delta \mathrm{t}$ function. The value corresponding to the $\mathrm{d}^{\prime}=1.0$ ordinate was taken as the threshold (i.e., the justnoticeable time increment) for a certain base interval $t$ and a particular stimulus parameter combination. These threshold time differences were plotted as a function of base time interval duration for all stimulus parameters; the outcome of such an analysis can be seen in Figures 2-4. Error bounds on the time difference thresholds were computed from the standard error of the mean around each observed $d^{\prime}$ value; their asymmetrical appearance reflects the nonlinearity of the $\mathrm{d}^{\prime}$ measure.

\section{RESULTS}

\section{Experiment 1: Discriminability of Time Intervals Marked by Tone Bursts of Different Intensities}

In Experiment 1, each time interval was marked by two $1-\mathrm{kHz}$ tone bursts. The second of the two markers was presented at a constant $86-\mathrm{dB}$ sound pressure level, whereas, the first marker had an intensity which varied from condition to condition. The difference $\Delta I$ between the two marker intensities was $0,30,40$, or $50 \mathrm{~dB}$. The stimulus diagram is illustrated in Figures 1a and $2 a$. Three subjects participated in the experiment.
Figures $1 \mathrm{~b}$ and $1 \mathrm{c}$ display performance (percent correct) as a function of base time interval duration for Subject 1 at three different $\Delta t$ values $(5,10$, and $20 \mathrm{msec}$-analogous to "one-step," "two-step," and "four-step" differences in traditional speech discrimination studies). The intensity difference $\Delta I$ is 0 in Figure $1 \mathrm{~b}$ and $50 \mathrm{~dB}$ in Figure 1c. While the essentially monotonic trend of the performance in Figure 1b may be contrasted with the slightly nonmonotonic shape of the curves in Figure 1c, the two sets of data are difficult to compare due to the farge differences in absolute performance level. For' this reason, we felt compelled to abandon this kind of data presentation and adopt another type, one that is embodied in all subsequent figures.

Figures $2 \mathrm{~b}, 2 \mathrm{c}$, and $2 \mathrm{~d}$ show time discrimination thresholds separately for the three subjects, whereas the same thresholds computed for their combined data are plotted in Figure 2e. Despite some individual differences, two common trends transpire. First, as the level of the first marker progressively decreases, so does discriminability of all time intervals. Second,

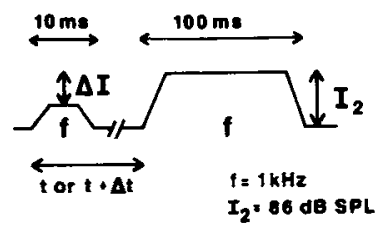

(a)

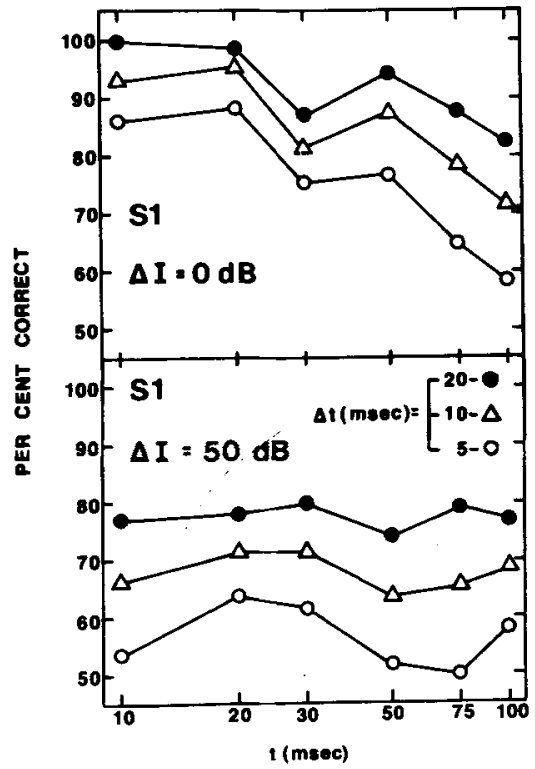

(b)

(c)

Figure 1. Results of Experiment 1 for Subject 1: The effect of intensity difference $(\Delta I)$ of two $1-\mathbf{k H z}$ tone burst markers on the discrimination of the time interval (t) between their onsets. The stimulus diagram is illustrated in Figure 1a. Results for $\Delta I=0$ are shown in Figure $1 \mathrm{~b}$ and for $\Delta I=\mathbf{5 0} \mathrm{dB}$ in Figure 1c. Abscissa: duration of the base interval t. Ordinate: performance level (percent correct). Parameter: time difference $\Delta t(5,10$, and 20 msec). 

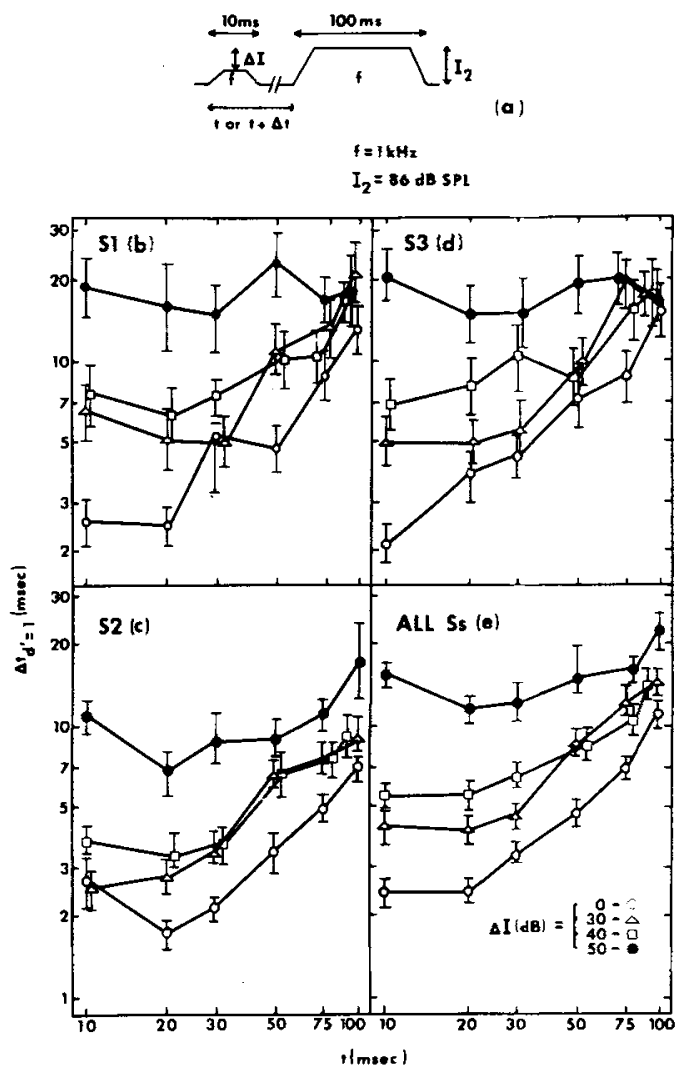

Figure 2. Results of Experiment 1: The effect of the intensity difference $(\Delta \mathrm{I})$ of two tone burst markers on the discrimination of the time interval $(t)$ between their onsets. Stimulus diagram is shown in Figure 2a, individual data of three subjects in Figures $2 b-2 d$, and combined results of all subjects in Figure $2 e$. Abscissa: duration of the base time interval t. Ordinate: time difference $\Delta t$ discriminable at the $d^{\prime}=1.0$ threshold level. Bars refer to \pm 1 standard error of the mean. Parameter: intensity difference $\Delta I=I_{2}-I_{1}$.

this performance decrement is relatively large for the shortest intervals as compared to the longer ones. In other words, the discrimination functions, monotonic at the $\Delta \mathrm{I}=0$ condition, ${ }^{3}$ became nonmonotonic at large intensity differences. The nonmonotonicity is most apparent in the average data where the variability is smallest. The relatively large variability in the data of the individual subjects, especially at the $\Delta \mathrm{I}=50 \mathrm{~dB}$ condition, reflects the considerable difficulty that the listeners encountered when simply trying to hear the first marker of the intervals.

\section{Experiment 2: Discriminability of Time Intervals Marked by Tone Bursts of Different Frequencies}

The objective of Experiment 2 was to examine the effect of a given difference between the frequencies of two tone-burst markers on the discriminability of the time interval enclosed by them. The level of all tone bursts was held constant at $86 \mathrm{~dB}$ SPL. The frequency of the first marker of each interval was always higher than, or equal to, that of the second marker. The geometric mean of the two marker frequencies was fixed at $1 \mathrm{kHz}$. A diagram of the stimulus is illustrated in Figure 3a. The effect of four frequency differences $\Delta \mathrm{f}$ was investigated: $0(1 \mathrm{kHz}$ both tones), $1 / 2$ octave $(1,189$ and $841 \mathrm{~Hz}), 1$ octave $(1,114$ and $707 \mathrm{~Hz})$, and 2 octaves $(2,000$ and $500 \mathrm{~Hz})$.
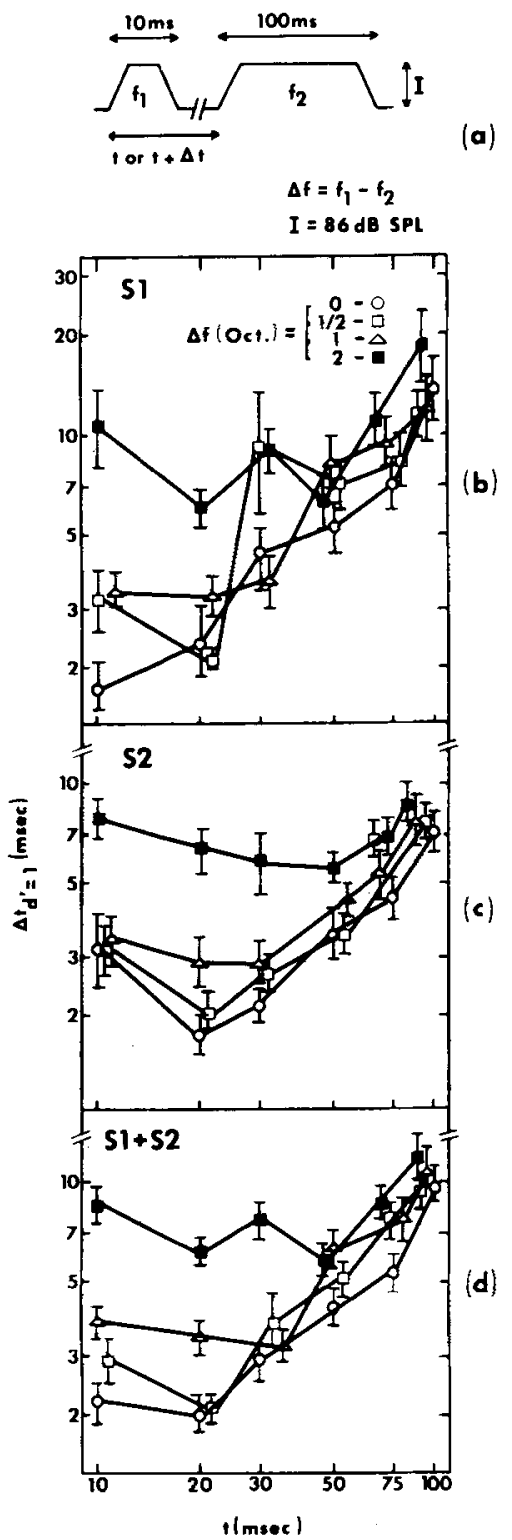

Figure 3. Results of Experiment 2: The effect of frequency difference ( $\Delta f)$ of the two tone burst markers on the discrimination of the time interval ( $t$ ) between their onsets. Figure 3a: stimulus diagram; Figures 3b-3c: results of two subjects; Figure 3d: combined results of the two subjects. Abscissa and ordinate as in Figure 2. Parameter is $\Delta f=f_{1}-f_{2}$ in octaves $\left[\left(f_{1} f_{2}\right)^{t / 2}=1 \mathrm{kHz}\right]$. 
Two subjects participated in this experiment-the third was unfortunately unable to complete his participation.

Results of the experiment are shown in Figures 3b and $3 c$ separately for the two subjects, and in Figure $3 \mathrm{~d}$ for their combined data. The figures depict time differences discriminable at threshold as a function of the base interval duration. Again, the performance of the two subjects was not exactly identical in this experiment, either. Nevertheless, the data suggest that frequency difference has no effect on time discrimination, as long as the intervals are relatively long $(>50 \mathrm{msec})$. On the other hand, discriminability of short time intervals (especially that of the 10 -msec interval) becomes poorer and poorer as the frequency difference between the markers increases from 0 to 2 octaves. At the $\Delta f=2$ octaves condition, the discrimination function for $10-100-\mathrm{msec}$ unfilled intervals has become nonmonotonic.

\section{Experiment 3: Discriminability of Time Intervals Marked by Tone Bursts of Unequal Durations}

In most previously reported experiments on the discrimination of unfilled auditory intervals (Abel, 1972; Chistovich, 1959; Divenyi \& Danner, 1977), the stimulus consisted of markers (noise bursts, tone bursts, or clicks) identical in duration. In these experiments, the time discrimination functions were found to be monotonic. In contrast, the stimulus of most experiments in which nonmonotonic time discrimination functions were observed employed dissimilar markers which differed also in their duration, the first marker being usually very brief (about $10 \mathrm{msec}$ ) and the second rather long (300-500 msec). To what extent could the nonmonotonic discrimination functions be attributed to the difference in marker duration? This question was addressed in Experiment 3 of the present study. Time intervals in this experiment were marked by pairs of $1-\mathrm{kHz}$ and $86-\mathrm{dB}$ SPL tone bursts. The duration of the first marker was fixed at $10 \mathrm{msec}$, whereas that of the second marker was 10 , 30,100 , or $300 \mathrm{msec}$, depending on the condition. A diagrammatic representation of the stimulus is shown in Figure 4a. Three subjects participated.

Results of the three individual subjects did not exhibit appreciable differences. Temporal discrimination functions obtained for the three subjects' combined data are displayed in Figure 4b. It appears that these functions are identical and essentially monotonic for all marker durations with one (minor) exception: the time discrimination threshold at the shortest $(10-\mathrm{msec})$ interval is somewhat higher when the second marker is very long $(300 \mathrm{msec})$. For one observer (Subject 2), however, such a slight increase in the threshold of the $10-\mathrm{msec}$ interval, as compared to that of the 20 -msec interval, could also be seen at the $\Delta \mathbf{I}=0$ condition in Experiment 1 (Figure 2c) and at the $\Delta \mathrm{f}=\mathbf{0}$ condition in Experiment 2 (Figure $3 \mathrm{c}$ ), in which the duration of the second marker was $100 \mathrm{msec}$.

\section{DISCUSSION}

When listeners are asked to discriminate time intervals that are marked by pairs of tone bursts similar in their intensities, frequencies, and duration, the just-noticeable time difference will be a monotonic function of the base interval. If, however, the two markers of an unfilled interval are considerably different in any of these three physical characteristics (especially the first two), the time discrimination function will become nonmonotonic. The essence of such a nonmonotonicity consists of the time discrimination thresholds being lowest in a certain temporal range and relatively higher for intervals that fall either above or below this range. It is not surprising that thresholds have been found to be larger for base intervals above the critical range (see right side of the curves in Figures 2, 3, and 4): such an observation is consistent with predictions of some monotonic psychophysical law of the sort we are accustomed to. (One such law, a modified form of Weber's law, was proposed by Divenyi \& Danner, 1977.) The threshold increase below the critical range represents a departure from such a psychophysical law (see left side of the curves in Figures 2, 3 , and 4). Since the magnitude of this increase is proportional to the extent to which the two markers are different in intensity, frequency, or duration, we may look for the origin of this threshold increase in perceptual interactions that arise when two different stimuli follow each other in rapid succession (Miller et al., 1976).

What are the specific interactions that are likely to

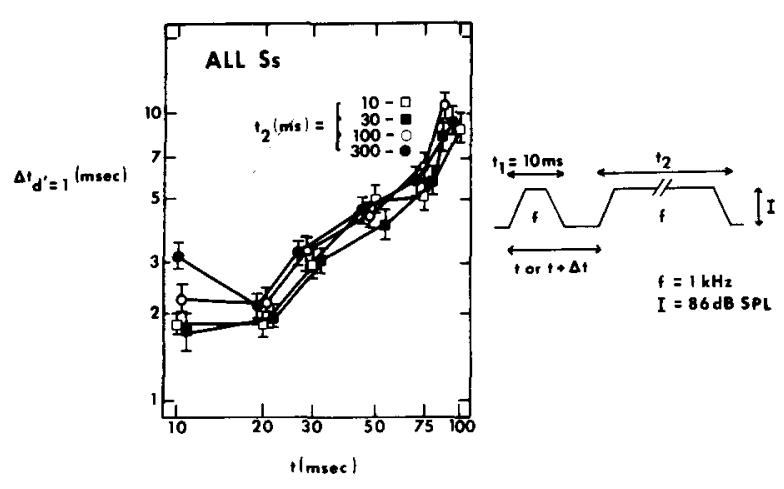

Figure 4. Results of Experiment 3: The effect of the duration of the second marker $\left(t_{2}\right)$ on the discrimination of the time interval (t) between two 1-kHz, 86-dB SPL tone burst markers. Figure 4a shows the stimulus diagram and Figure $4 b$, the combined results of three subjects. Abscissa and ordinate as in Figure 2. Parameter is $t_{2}$. 
cause interference in the discrimination of time intervals defined by two tonal markers close in time? Results of the present experiments suggest two probable sources. The first of these is backward masking: Experiment 1 (and Experiment 3, albeit only to a very slight extent) demonstrated that, when the interval to be discriminated is very short, the same parameter variations that result in increasing backward masking also produce increasing interference in time discrimination. In Experiment 1, it was shown that a marked decrement in the intensity of the first two $1-\mathrm{kHz}$ tone bursts decreases, and eventually disrupts, discriminability of the time interval that they enclose. Results of Experiment 3 showed that we can expect at least some decrease in temporal discriminability even when the two markers are equally intense, provided that the duration of the second marker is disproportionately long with respect to that of the first. These two factors, intensity difference and duration difference, are known to cause backward masking (Elliott, 1971). Nevertheless, we are not suggesting here that the first marker should be entirely obliterated by the second before listeners encounter difficulty in accurately perceiving the length of the time interval between the two markers. Since, despite a few attempts to study the problem (Abel, 1972; Creelman, 1962; Divenyi \& Danner, 1977), it is still not exactly known what part of the auditory stimulus acts as a time marker, we can only infer from our data that the effectiveness of the first marker of a short interval will be reduced whenever it is likely to undergo a certain degree of backward masking, i.e., whenever it is short and soft and whenever it is followed by a long and loud second marker. In other words, the listener will accurately perceive a time interval defined by two tones only when he can adequately hear both of them-a conclusion hardly unexpected. ${ }^{4}$

The results of Experiment 2, however, suggest that there must be a second perceptual interaction that interferes with the discrimination of short intervals, an interaction complementing backward masking. Indeed, it is known that backward masking diminishes as the difference between masker frequency and probe frequency increases (Samoilova, 1960); yet, data of Experiment 2 indicate that discrimination of a 10 -msec interval gradually worsens as the difference between the two marker frequencies increases. Thus, these data suggest that it is a process analogous to auditory stream segregation (Bregman \& Campbell, 1971), rather than backward masking, that must operate in those situations where the markers of a brief time interval are likely to be processed in two separate channels. In fact, it has been noted by several workers (Williams \& Perrott, 1972; van Noorden, Note 1) that auditory time perception becomes less accurate when the intervals are marked by tones widely separated in frequency. The present results may be regarded as another instance illustrating the same phenomenon. While the effect of frequency difference seems to disappear for longer time intervals, it is definitely an important factor for short ones. This finding suggests that short auditory intervals may be accurately perceived only if the markers are close in frequency, possibly within the same critical band.

It is not unreasonable to suppose that these two interactions, i.e., backward masking and auditory stream segregation, should also play a role in the perception of time intervals where the markers are two distinct, complex sounds widely different in their spectrum, intensity, and duration. A good example for temporal perception in such complex sounds is the perception of voicing in stop consonant-vowel syllables-a process in which accurate resolution of the voice onset time plays a key role. The question that arises is: what strategy or strategies must a listener follow if he is to accurately resolve short VOT intervals? Assuming that he is able to monitor at most a finite number of channels (i.e., spectral regions) of a complex sound input, and that he is unable to integrate temporal information across channels (Pisoni, 1977), his options as to the strategy to follow are quite limited. As one strategy, he may choose to listen only in one band-in which case the first marker (i.e., the plosive burst) will undergo a certain amount of backward masking, due to its temporal closeness to the more intense and longer second marker (i.e., the vowel sound analyzed in the same band)-and he will lose temporal resolving power. Alternatively, he may adopt the strategy of listening to the plosive burst in one band and to the vowel sound in another, with the aim of minimizing backward-masking effects. ${ }^{5}$ In fact, he may be able to achieve this by finding for the first marker a certain band in which the burst energy is relatively large and the vowel energy is relatively low, and for the second marker another, different band in which the vowel energy is large and the burst energy is negligible. Nevertheless, such "band-hopping" might not be the best way for him to improve his temporal resolving capability: whatever advantage he may gain by successfully avoiding backward masking, he would lose it due to a reduction of his ability to perceptually organize two auditory events that are processed in two different streams. Thus, no matter what strategy the observer follows, he is caught in the snare of either one or the other of two perceptual interactions, both of which will impair his ability to resolve short VOTs. However, as the VOT of the speech sound increases, so does his relative accuracy to perceive it correctly, because of the diminished role of both backward masking (Samoilova, 1960) and auditory stream segregation (Bregman \& Campbell, 1971) at larger temporal separations 
between two sounds. Ultimately, at some critical VOT duration, both perceptual interactions will eventually vanish, for all practical purposes. However, if VOT is increased beyond such a critical duration, temporal resolution will again worsen-this time as a result of the psychophysical law that governs, in general, discrimination of all auditory time intervals. Thus, a stimulus-bound explanation for the nonmonotonicity found in VOT discrimination functions can be directly derived from the present results.

However, there are three caveats pertaining to such an extension of the present data to VOT perception, caveats of which the reader should be aware. First, the nonmonotonic discrimination functions which we obtained for time intervals marked by tone bursts do not show the sharp, V-shaped nonmonotonicity seen in VOT discrimination functions (see Miller et al., 1976). So far, we have been unable to come across a combination of sinusoidal stimuli which would have yielded the sharp discontinuity we had hoped for. ${ }^{6} \mathrm{We}$, therefore, do not consider as completely unlikely the possibility that phonemic reference in speech and speech-like stimuli, as well as the underlying perceptual categories, could help reinforce the discontinuity in the time discrimination functions. Second, we would like to stress that our subjects were well (if not overly well) trained. It has been shown (Samuel, 1977) that training does alter the shape of VOT discrimination functions. Had it been possible to collect reliable data from nonpracticed observers in our time discrimination experiments, we would have done so and, possibly, would have obtained much sharper discontinuities. ${ }^{7}$ Third, it has been shown (Macmillan et al., 1977; Sachs \& Grant, 1976) that the psychophysical method and the stimulus uncertainty it entails also affect the shape of the discrimination function. A low stimulus-uncertainty situation seems to yield a flatter VOT-discrimination function for synthetic speech stimuli than do high stimulus-uncertainty paradigms. Much of the classical results on VOT discrimination (e.g., Abramson \& Lisker, 1970) were obtained using a high-uncertainty, roving-standard ABX method. A single-level 2AFC paradigm, by definition, would have yielded optimum performance for any given parameter combination. Our four-level 2AFC paradigm, though still on the low-uncertainty side, represents a compromise between these two extreme cases.

These reservations notwithstanding, we believe that the similarities between our results and VOT discrimination data reported by other workers are quite suggestive. Although we are unwilling to make inferences as to the special or less-than-special nature of speech perception at this point, we hope that we have come closer to understanding certain stimulus factors that influence VOT perception.

\section{REFERENCE NOTES}

1. van Noorden, L.P.A.S. Discrimination of time intervals bounded by tones of different frequencies. Instituut voor Perceptie Onderzoek (Eindhoven, the Netherlands), Annual Progress Report, 1971, 6, 12-15.

2. Fujisaki, H., \& Kawashima, T. Some experiments on speech perception and a model for the perceptual mechanism. Annual Report of the Engineering Research Institute, Vol. 29, Faculty of Engineering, University of Tokyo, Tokyo, 1970, 207-214.

\section{REFERENCES}

ABEL, S. M. Discrimination of temporal gaps. Jourmal of the Acoustical Society of America, 1972, 52, 519-524.

Abramson, A. S., \& Lisker, L. Discrimination along the voicing continuum: Cross-language tests. In Proceedings of the Sixth Intemational Congress of Phonetic Sciences, Prague, 1967. Prague: Academia Publishing House of the Czechoslovak Academy of Science, 1970. Pp. 569-573.

Bregman, A. S., \& Campbell, J. L. Primary auditory stream segregation and perception of order in rapid sequence of tones. Journal of Experimental Psychology, 1971, 89, 244-249.

Chistovich, L. A. Discrimination of the time interval between two short acoustic pulses. Soviet Physics: Acoustics, 1959, 5, 493-496.

Creelman, C. D. Human discrimination of auditory duration. Journal of the Acoustical Society of America, 1962, 34, 582-593.

Divenyi, P. L., \& DanNer, W. F. Non-monotonic discrimination functions for time intervals: Implications for VOT perception. Journal of the Acoustical Society of America, 1975, 58, S36(A).

DivenY, P. L., \& DANNER, W. F. Discrimination of time intervals marked by brief acoustic pulses of various intensities and spectra. Perception \& Psychophysics, 1977, 21, 125-142.

Elliot, L. L. Backward and forward masking. Audiology, 1971, 10, 65-76.

GREEN, D. M., \& SwETs, J. A. Signal detection theory and psychophysics. New York: Wiley, 1966.

HIRSH, I. J. Auditory perception of temporal order. Journal of the Acoustical Society of A merica, 1959, 31, 757-767.

Liberman, A., Cooper, F. S., Shankweiler, D., \& StuddertKennedy, M. Perception of the speech code. Psychological Review, 1967, 74, 431-461.

Lisker, L., \& Agramson, A. The voicing dimension: Some experiments in comparative phonetics. In Proceedings of the Sixth Intermational Congress of Phonetic Sciences, Prague, 1967. Prague: Academia Publishing House of the Czechoslovak Academy of Science, 1970. Pp. 563-568.

Macmillan, N. A., Kaplan, H. L., \& Creelman, C. D. The psychophysics of categorical perception. Psychological Review, 1977, 84, 452-471.

Miller, J. D., Pastore, R. E., Wier, C. C., Kelly, W. J., \& Dooling, R. J. Discrimination and labeling of noise-buzz sequences with varying noise-lead times: An example of categorical perception. Journal of the Acoustical Society of America, 1976, 60, 410-417.

Penner, M. J. The effect of marker variability on the discrimination of temporal intervals. Perception \& Psychophysics, 1976, 19, 466-469.

Pisoni, D. B. Identification and discrimination of the relative onset time of two component tones: Implications for voicing perception. Journal of the Acoustical Society of America, 1977, 61, 1352-1361.

SAMOILOVA, I. K. Investigation of minimum perceptible intervals between two tone signals. Soviet Physics: Acoustics, 1960, 6, 382-388.

SAMUEL, A. G. The effect of discrimination training on speech perception: Noncategotical perception. Perception \& Psychophysics, 1977, 22, 321-330.

SACHS, R. M., \& GRANT, K. Stimulus correlates in the perception 
of voice onset sime (VOT): 11. Discrimination of speech with high and low stimulus uncertainty. Journal of the Acoustical Society of America, 1976, 60, S91.

van Noorden, L.P.A.S. Minimum differences of level and frequency for perceptual fission of tone sequences $\mathrm{ABAB}$. Journal of the Acoustical Society of America, 1977, 61, 1041-1045.

Williams, K. N., \& PERRotr, D. R. Temporal resolution of tonal pulses. Journal of the Acoustical Society of America, 1972, 51, 644-647.

\section{NOTES}

1. We are referring to some generalized form of Weber's law, such as Stevens' law, for example.

2. It has been proposed (e.g., Fujisaki \& Kawashima, Note 2) that part of the explanation for nonmonotonic discrimination functions in speech perception may be found in the increased memory load that stimuli delivered according to the conventionally used ABX paradigm represent. Results of the Sachs and Grant experiment suggest that for trained listeners this memory factor may not play any important role.

3. Subject 2 behaved in a way different from the generally monotonic trend: his threshold at $t=10 \mathrm{msec}$ was somewhat higher than at $t=20 \mathrm{msec}$ in the $\Delta \mathrm{I}=0$ condition. A possible explanation for this irregularity is given in the section describing the results of Experiment 3.
4. One reviewer suggested that another factor, different from backward masking, may have intervened to produce the nonmonotonic shape of the discrimination functions shown in Figure 2. The factor suggested is auditory stream segregation on the basis of intensity differences (van Noorden, 1977). While such an interpretation remains possible, one has to bear in mind that, at some of the intensity differences used in the present experiment, the first markers of each interval were extremely faint, if not completely inaudible. Stream segregation for such extreme stimulus levels has not yet been demonstrated.

5. A previous study (Divenyi \& Danner, 1975) showed that $1 / 3$-octave filtered noise-buzz sequences (originally filtered such that they followed the spectral characteristics of a transitionless $/ \mathrm{t} /-/ \mathrm{a} / \mathrm{CV}$ pair) give rise to nonmonotonic time discrimination functions. In that study, we were also able to notice the presence of a perceptual interference having a magnitude proportional to the difference between the intensity of the noise and that of the buzz inside a particular $1 / 3$-octave band.

6. The combination that seemed to offer some possibility was the one in which a short $1-\mathrm{kHz}$ burst was followed by a long $1-\mathrm{kHz}$ burst amplitude-modulated at a $100-\mathrm{Hz}$ rate.

7. Unfortunately, time discrimination studies require a great deal of training - and sometimes even a lengthy training is insufficient to achieve stable performance (M. J. Penner, personal communication).

(Received for publication January 17, 1978; revision accepted August 11, 1978.) 